\title{
NRF2-Independent Regulation of Intestinal Constitutive Androstane Receptor by the Pro-Oxidants Cadmium and Isothiocyanate in hUGT1 Mice ${ }^{\mathbb{}}$
}

\author{
Miles Paszek and Robert H. Tukey \\ Laboratory of Environmental Toxicology, Departments of Chemistry and Biochemistry (M.P.) and \\ Pharmacology (R.H.T.), University of California, San Diego, La Jolla, California
}

Received September 25, 2019; accepted November 1, 2019

\begin{abstract}
Environmental toxicants such as heavy metals from contaminated water or soil and isothiocyanates (ITC) from dietary sources act as pro-oxidants by directly generating reactive oxygen species (ROS) or through depleting cellular antioxidants such as glutathione. Toxicants can alter drug metabolism, and it was reported that CYP2B10 and UGT1A1 are induced by phenethyl isothiocyanate (PEITC) through the constitutive androstane receptor (CAR). The possibility that nuclear factor erythroid 2-related factor 2 (NRF2), the master regulator of the antioxidant response, could coactivate CAR was investigated in neonatal hUGT1/Nrf2 ${ }^{-1}$ mice. Neonatal mice were treated with PEITC or cadmium $\left(\mathrm{Cd}^{2+}\right)$ by oral gavage for 2 days. Both PEITC and $\mathrm{Cd}^{2+}$ induced UGT1A1 RNA and protein in intestinal tissues in both $h U G T 1 / N_{r f 2}{ }^{+/-}$and $h U G T 1 / N_{r f 2}{ }^{-/-}$neonates, indicating NRF2independent regulation of UGT1A1. Increases in CYP2B10 RNA in intestinal tissues were observed following PEITC or $\mathrm{Cd}^{2+}$ exposure. Activation of intestinal CAR by $\mathrm{Cd}^{2+}$ exposure was directly assessed
\end{abstract}

by nuclear fractionation and Western blot analyses at $0.5,1,2$, and 4 hours after treatment in hUGT1 neonates and after 48 hours in hUGT1/Nrf2 ${ }^{+/-}$and hUGT1/Nrf2 ${ }^{-/-}$neonates. CAR localized to the nucleus independently of NRF2 $\mathbf{4 8}$ hours after exposure. Substantial CAR localization to the nucleus occurred at the 2- and 4-hour time points, coinciding with a decrease in phosphorylation of cytoplasmic extracellular signal-regulated kinases 1 and 2 and a nuclear increase in P38/p-P38 content. This suggests that a novel oxidative stress-MAPK-CAR axis exists with phenotypic consequences.

\section{SIGNIFICANCE STATEMENT}

Pro-oxidant toxicants can alter drug metabolism through activation of CAR, independent of the NRF2-KEAP1 signaling pathway. Changes in proteins associated with drug metabolism and linked to increases in intestinal maturation are mediated through an oxidative stress-MAPK-CAR axis.

\section{Introduction}

Exposure to certain environmental toxicants is capable of altering drug metabolism through the activation of selective transcription factors such as xenobiotic and nuclear receptors (XRs and NRs). The activation of XRs and NRs typically occurs through direct ligand binding to the receptor or through post-translation modifications. In vitro models enable medium- to high-throughput screening of the abilities of compounds to activate nuclear receptors, whereas in vivo models provide more complex details such as absorption, elimination, and tissue-specific metabolism.

An in vivo model that lends itself well to reverse genetic approaches is the humanized UDP-glucuronosyltransferase 1 ( $h U G T 1$ ) mouse model owing to the readily quantifiable phenotype that changes following XR

This work was supported by U.S. Public Health Service Grants from the National Institutes of Health National Institutes of Environmental Health Sciences [ES010337] and General Medical Sciences [GM126074] and the UCSD Graduate Training Program in Cellular and Molecular Pharmacology [GM007752].

https://doi.org/10.1124/dmd.119.089508.

SThis article has supplemental material available at dmd.aspetjournals.org. or NR activation (Fujiwara et al., 2010; Chen and Tukey, 2018). The phenotype is presented as neonatal hyperbilirubinemia, a condition unique to humans in which increased turnover of red blood cells causes heme degradation to bilirubin that accumulates systemically (Pearson, 1967). The accumulation of bilirubin occurs because hepatic expression of UGT1A1, the only enzyme capable of conjugating bilirubin for excretion (Bosma et al., 1994), is developmentally delayed at the transcriptional level (Burchell et al., 1989). However, we have demonstrated that control of intestinal UGT1A1 during development plays a key role in the clearance of serum bilirubin during the neonatal period (Fujiwara et al., 2010; Liu et al., 2016; Chen et al., 2017). Induction of the UGT1Al gene in $h U G T 1$ mice can be mediated by multiple XRs and NRs, such as the pregnane X-receptor (PXR) (Chen et al., 2012), constitutive androstane receptor (CAR) (Sugatani et al., 2001), and peroxisome proliferator-activated receptor $\alpha$ (PPAR $\alpha$ ) (Senekeo-Effenberger et al., 2007), as well as environmental toxicant sensors such as the aryl hydrocarbon receptor (AhR) (Yueh et al., 2005; Bonzo et al., 2007) and the nuclear factor erythroid 2-related factor 2 (NRF2) (Yueh and Tukey, 2007). Following exposure, activation of one or more of these XRs and NRs induces UGT1A1 gene expression

ABBREVIATIONS: AhR, aryl hydrocarbon receptor; ANOVA, analysis of variance; CAR, constitutive androstane receptor; ERK1/2, extracellular signal-regulated kinases 1 and 2; hUGT1, humanized UDP-glucuronosyltransferase 1; ITCs, isothiocyanates; KEAP1, Kelch-like ECH-associated protein 1; MAPK, mitogen-activated protein kinase; NRF2, nuclear factor erythroid 2-related factor 2 (nuclear factor erythroid-derived 2-like 2); NRs, nuclear receptors; P38, mitogen-activated protein kinase 11; PCR, polymerase chain reaction; PEITC, phenethyl isothiocyanate; ROS, reactive oxygen species; RT-qPCR, reverse transcription-quantitative PCR; TSB, total serum bilirubin; XRs, xenobiotic receptors NRs nuclear receptors. 
resulting in a reduction of total serum bilirubin (TSB) levels in $h U G T 1$ mice (Yueh et al., 2017; Chen and Tukey, 2018). Known transcriptional targets can be examined to determine which XR or NR is inducing UGT1A1; for example, CAR induces the Cyp 2 b10 (Honkakoski et al., 1996) gene, whereas activated NRF2 can induce genes such as Gsta1/2 (McMahon et al., 2001), which are sensitive to oxidative stress. The receptor-mediated induction of UGTIAl can be confirmed in neonatal $h U G T 1$ mice that express a null allele for the $X R$ - or $N R s$, where elevated TSB levels following exposure can be directly linked to a dependency on the deleted receptor (Chen et al., 2013; Liu et al., 2016; Yoda et al., 2017).

Recently, it was reported that the dietary pro-oxidants isothiocyanates (ITCs) induce hepatic UGT1A1 in a CAR-dependent fashion (Yoda et al., 2017). This is significant because the mechanisms of direct and indirect activation of CAR are well characterized (Yoshinari et al., 2003; Osabe and Negishi, 2011; Mutoh et al., 2013; Timsit and Negishi, 2014) but have never directly implicated oxidative stress as a CAR activator. Additionally, other environmentally relevant toxicants, such as cadmium $\left(\mathrm{Cd}^{2+}\right)$ and arsenic $\left(\mathrm{As}^{3+}\right)$ that increase reactive oxygen species (ROS) have been reported to induce intestinal Cyp2b10 and UGT1Al gene expression in $h U G T 1$ mice (Fujiwara et al., 2010; Yoda et al., 2017). However, UGT1A1 induction by pro-oxidants has been linked to NRF2 (Yueh and Tukey, 2007), the master regulator of the antioxidant response (Moi et al., 1994). The induction mechanism of intestinal UGT1A1 in $h U G T 1$ mice by agents such as $\mathrm{Cd}^{2+}$ and ITCs were examined with respect to their link toward activation of NRF2 and CAR.

\section{Materials and Methods}

Animal Studies. All animals were housed at the University of California San Diego Animal Care Facility with access to food and water ad libitum. All animal use including experimental procedures, handling, and protocols were approved by the UCSD Animal Care and Use Committee (IACUC) and adhere to the National Institutes of Health Guide for the Care and Use of Laboratory Animals. UCSD's Animal Care Program is accredited by the Association for the Assessment and Accreditation of Laboratory Animal Care (AAALAC). Humanized $U G T 1$ ( $h U G T 1$ ) mice were previously generated as described. $N r f 2^{-\prime-}$ mice were purchased from Jackson Laboratory (stock no: 017009), and $h U G T 1 / \mathrm{Nrf}^{+/-}$mice were generated and crossed for comparison with $h U G T 1 / N r f 2^{+/+}$mice, utilizing both males and females. Additional studies involved breeding hUGT1/Nrf2 ${ }^{+/-}$with hUGTl/ $\mathrm{Nrf} 2^{-1-}$ to generate male and female mice used for neonatal studies. Neonates were treated on day 10 and 11 with $\mathrm{CdCl}_{2} \cdot 2-1 / 2 \mathrm{H}_{2} \mathrm{O}\left(\mathrm{Cd}^{2+}\right)$ or $\mathrm{NaAsO}_{2}\left(\mathrm{As}^{3+}\right)$ (Sigma-Aldrich) at $10 \mathrm{mg} / \mathrm{kg}$ or PEITC (Sigma Aldrich) at $200 \mathrm{mg} / \mathrm{kg}$ by oral gavage or with water or corn oil (5 $\mu \mathrm{l} / \mathrm{g}$ of bodyweight) for vehicle control. The dosages for $\mathrm{Cd}^{2+}$ and PEITC treatments were selected from dose-response curves where both the greatest reduction in total serum bilirubin levels and no observable behavioral or gross anatomic phenotypes of toxicity were obtained. Animals were anesthetized with isoflurane and euthanized by cervical dislocation.

Real-Time Polymerase Chain Reaction. Intestines were collected and briefly washed with ice-cold phosphate-buffered saline (PBS) three times before being snap frozen in liquid nitrogen. Livers were collected and washed once with ice-cold PBS before snap freezing in liquid nitrogen. RNA was extracted with TRIzol Reagent (ThermoFisher Scientific) as described by the manufacturer's protocol. cDNA was synthesized using iScript cDNA Synthesis Kit (Bio-Rad) according to the manufacturer's protocol. Real-time polymerase chain reaction (PCR) was performed using a CFX96 Real-Time PCR Detection system (BioRad) and SSoAdvanced Universal SYBR Green Supermix (Bio-Rad) as described by the manufacturer's protocol. All values represent independent biologic replicates and technical singlets as analyzed by the $\Delta \Delta C T$ method. Primers used for real time PCR analysis of selected genes can be found in Table 1.

Western Blot Analysis. Whole lysate was obtained from homogenization of pulverized intestinal tissues in RIPA buffer (cat. no. 20-188; Millipore Sigma) containing protease (cat. no. 78429; ThermoFisher Scientific) and phosphatase (cat. no. 78420; ThermoFisher Scientific) inhibitors. Pulverized tissues in lysis buffer $(1: 4 \mathrm{wt} / \mathrm{vol})$ were blade homogenized and incubated on ice for 15 minutes before centrifugation $\left(10,000 \mathrm{~g}, 4^{\circ} \mathrm{C}, 20\right.$ minutes) and supernatant storage at $-80^{\circ} \mathrm{C}$.
Nuclear fractionation used a two-buffer extraction. Buffer A contained $10 \mathrm{mM}$ HEPES, $1.5 \mathrm{mM} \mathrm{MgCl} 2,10 \mathrm{mM} \mathrm{KCl}, 0.5 \mathrm{mM}$ dithiothreitol (DTT), and $0.05 \%$ Igepal at $\mathrm{pH} 7.9$ with protease and phosphatase inhibitors. Buffer B contained $5 \mathrm{mM}$ HEPES, $1.5 \mathrm{mM} \mathrm{MgCl} 2,0.2 \mathrm{mM}$ EDTA, $0.5 \mathrm{mM}$ DTT, and $26 \%$ glycerol (v/v) at $\mathrm{pH} 7.9$ with protease and phosphatase inhibitors. Pulverized tissues were homogenized in buffer A (1:4 wt/vol) using a Potter-Elvehjem homogenizer for 10 passes. Homogenates were incubated on ice for 10 minutes prior to centrifugation $\left(1500 \mathrm{~g}, 4^{\circ} \mathrm{C}, 10\right.$ minutes). The supernatant containing the cytosol was stored at $-80^{\circ} \mathrm{C}$. The pellet containing the nuclear contents was washed with $200 \mu \mathrm{l}$ of buffer A before resuspension in $400 \mu \mathrm{l}$ of buffer B. After resuspension, $\mathrm{NaCl}$ was added to a final concentration of $300 \mathrm{mM}$ and the solution was sonicated for 5 seconds and this repeated three times. Samples were incubated on ice for 30 minutes before centrifugation $\left(21,000 \mathrm{~g}, 4^{\circ} \mathrm{C}, 20\right.$ minutes). The supernatant containing the nuclear fraction was stored at $-80^{\circ} \mathrm{C}$. Protein concentration was determined via Bradford assay (Bio-Rad), and BSA (New England BioLabs) protein standards were created for simple linear regressions.

Samples were run on NuPAGE 4\%-12\% Bis-Tris Protein Gels (Invitrogen) before transfer to polyvinylidene difluoride membranes. Membranes were blocked with 5\% nonfat milk or BSA for phospho-antibodies in TBST and incubated with primary antibodies overnight at $4^{\circ} \mathrm{C}$ and secondary antibodies for 1 hour at room temperature. Imaging was performed on a ChemiDoc Touch imaging system (BioRad) using Clarity Western ECL substrate (Bio-Rad). All antibodies used for protein expression and localization analyses can be found in Table 2. All samples represent biologic replicates and technical singlets.

Bilirubin Measurement. Approximately $50 \mu \mathrm{l}$ of blood was obtained from the submandibular vein. Blood was centrifuged for 2 minutes at 16,000g, and the supernatant was measured using a Unistat Bilirubinometer (Reichert Inc.) to determine total serum bilirubin levels.

Statistical Analysis. All statistical analyses were performed using GraphPad Prism's (V 6.07) two-way analysis of variance (ANOVA) with multiple comparisons. Averages \pm S.E.M. are shown. Differences were considered statistically significant when $P<0.05$. Statistical significance is represented by the following: $* P<0.05$, $* * P<0.01, * * * P<0.001, * * * * P<0.0001$.

\section{Results}

$\mathrm{Cd}^{2+}$ Treatment Induces Oxidative Stress Markers in an NRF2-Dependent Manner. Intestinal tissues were collected from hUGT1/Nrf2 ${ }^{+/+}, h U G T 1 / \mathrm{Nrf}^{+/-}$, and $h U G T 1 / \mathrm{Nrf}^{-/-}$neonates treated orally with $10 \mathrm{mg} / \mathrm{kg} \mathrm{Cd}^{2+}$, and gene targets known to be induced by NRF2 in response to oxidative stress examined by reverse transcriptionquantitative PCR (RT-qPCR) (Fig. 1). Statistically significant inductions were detected in $\mathrm{Cd}^{2+}$-exposed $h U G T 1 / \mathrm{Nrf}^{+/+}$and $h U G T 1 / \mathrm{Nrf}^{+/-}$ neonates for Nqol (2.7- and 1.7-fold), Ho-1 (6.8- and 4.3-fold), Gstal (37.8- and 33.0-fold), Gsta2 (9.3- and 7.9-fold), and Gstm3 (15.8- and 8.7-fold) genes. A gene-dosage effect comparing responses between hUGT1 and hUGT1/Nrf2 ${ }^{+/}$neonatal mice was observed for the induction of the Nqo1, Ho-1, and Gstm3 genes. This indicates haploinsufficiency related to NRF2's induction of antioxidant-response genes. When we examined the induction of these genes in $h U G T 1 / \mathrm{Nrf}^{-/-}$mice following $\mathrm{Cd}^{2+}$ treatment, there was no statistically significant difference from the vehicle treatment of $h U G T 1$ mice, confirming that the induction of these genes in the gastrointestinal tract originates from the generation of ROS in the intestines. With oral $\mathrm{Cd}^{2+}$ treatment, there was no induction of these genes in hepatic tissue.

$\mathrm{Cd}^{2+}$ Induces Intestinal UGT1A1 and Lowers TSB Levels in an NRF2-Independent Fashion. It was previously determined that oral $\mathrm{Cd}^{2+}$ treatment of neonatal $h U G T 1$ mice led to the induction of intestinal UGT1A1 (Liu et al., 2016). To determine if induction of the intestinal UGT1A1 gene by $\mathrm{Cd}^{2+}$ was dependent on the generation of ROS and activation of the NRF2-Kelch-like ECH associated protein 1 (KEAP1) pathway, $h U G T 1 / \mathrm{Nrf}^{+/-}$mice were mated and 10-day old newborns treated with $10 \mathrm{mg} / \mathrm{kg} \mathrm{Cd}^{2+}$ or water by oral gavage once per day for 2 days before blood and intestinal tissue were collected. With $h U G T 1$, hUGT1/Nrf2 $2^{+/-}$, and $h U G T 1 / \mathrm{Nrf}^{-/-}$mice, $\mathrm{Cd}^{2+}$ treatment eliminated 
TABLE 1

Primers used for real-time PCR analysis of selected genes

\begin{tabular}{|c|c|c|}
\hline Gene & Forward $\left(5^{\prime} \rightarrow 3^{\prime}\right)$ & Reverse $\left(5^{\prime} \rightarrow 3^{\prime}\right)$ \\
\hline hUGT1A1 & cca tca tgc cca ata tgg $\mathrm{tt}$ & cca caa ttc cat gtt ctc ca \\
\hline Cyplal & TGC CCT TCA TTG GTC ACA TG & CAC GTC CCC ATA CTG CTG ACT \\
\hline Cyp $2 b 10$ & AAG GAG AAG TCC AAC CAG CA & CTC TGC AAC ATG GGG GTA CT \\
\hline Cyp3a11 & ACA AAC AAG CAG GGA TGG AC & CCC ATA TCG GTA GAG GAG CA \\
\hline Cyp $4 a 10$ & CAC ACC CTG ATC ACC AAC AG & TCC TTG ATG CAC ATT GTG GT \\
\hline Nqo1 & TTT AGG GTC GTC TTG GCA AC & GTC TTC TCT GAA TGG GCC AG \\
\hline Ho-1 & ACA GGG TGA CAG AAG AGG CTA AGA C & ATT TTC CTC GGG GCG TCT CT \\
\hline Gstal & AGC CCG TGC TTC ACT ACT TC & TCT TCA AAC TCC ACC CCT GC \\
\hline Gsta2 & AAT CAG CAG CCT CCC CAA T & TCC ATC AAT GCA GCC ACA CT \\
\hline Gstm3 & AAA CCT GAG GGA CTT CCT GG & AAC ACA GGT CTT GGG AGG AA \\
\hline$I l-1 b$ & GCA ACT GTT CCT GAA CTC AAC & ATC TTT TGG GGT CCG TCA ACT \\
\hline Il-6 & ACC AGA GGA AAT TTT CAA TAG GC & TGA TGC ACT TGC AGA AAA CA \\
\hline $\operatorname{Tnf} \alpha$ & GAT CGG TCC CCA AAG GGA TG & GGC TAC AGG CTT GTC ACT CG \\
\hline Glbl & CAC TGC TGC AAC TGC TGG & ATG TAT CGG AAT GGC TGT CC \\
\hline $\operatorname{Lrp} 2$ & CCA GGA TTC TGG TGA TGA GG & CGG GAA CTC CAT CAC AAA CT \\
\hline Krt20 & CCT GCG AAT TGA CAA TGC TA & CCT TGG AGA TCA GCT TCC AC \\
\hline Sis & ACC CCT AGT CCT GGA AGG TG & CAC ATT TTG CCT TTG TTG GAT GC \\
\hline Cph & CAG ACG CCA CTG TCG CTT & TGT CTT TGG AAC TTT GTC TGC \\
\hline
\end{tabular}

circulating TSB levels within 2 days, indicating that a mechanism had been activated to induce intestinal UGT1A1 (Fig. 2A). Similar reductions were observed for PEITC- and $\mathrm{As}^{3+}$-treated neonatal mice (Supplemental Fig. 1). Analysis of UGT1A1 expression at both the gene and protein levels firmly establish that $\mathrm{Cd}^{2+}$ exposure induces intestinal UGT1A1 (Fig. 2, B and C). Unlike the $\mathrm{Cd}^{2+}$-initiated induction of the Nqol, Ho-1, Gstal, Gsta2, and Gstm3 genes (Fig. 1), which are dependent on NRF2, induction of the intestinal UGT1Al gene by $\mathrm{Cd}^{2+}$ is not linked to the NRF2-KEAP1 pathway. It should be noted that an antioxidant-response element (ARE) has previously been identified flanking the UGTIAl gene in the phenobarbital-response enhancer module region (PBREM), and has been demonstrated to bind the antioxidant tert-butylhydroquinone (tBHQ)-inducible NRF2 in treated HepG2 cells (Yueh and Tukey, 2007). In addition, the intraperitoneal administration of tBHQ to adult TgUGT1 mice led to induction of intestinal UGT1A1, implicating the NRF2-KEAP1 pathway as a regulator of the UGT1A1 gene (Yueh and Tukey, 2007). However, the oral administration of $\mathrm{Cd}^{2+}$ and induction of intestinal UGT1A1 in $h U G T 1$ neonatal mice does not require NRF2-KEAP1 signaling.

Intestinal Cyp2b10 Is Positively Regulated by $\mathrm{Cd}^{2+}$ Exposure and Induces Nuclear Accumulation of CAR. The nuclear receptors that induce UGT1A1 were examined by RT-qPCR analysis for activation of their known target genes (Supplemental Fig. 2). Only CYP2B10 was substantially induced by $\mathrm{Cd}^{2+}$ in $h U G T 1 / \mathrm{Nrf2} 2^{+/+}$ (8.4-fold), hUGT1/Nrf2 $2^{+/-}$(13.5-fold), and hUGT1/Nrf2 $2^{-/-}$(9.8-fold) neonates; however, only heterozygotes reached statistical significance

TABLE 2

Antibodies used for Western blot analysis

\begin{tabular}{llc}
\hline Antibody & \multicolumn{1}{c}{ Supplier } & Product No. \\
\hline Anti-mouse IgG, HRP-linked & Cell Signaling Technology & $\# 7076$ \\
Anti-rabbit IgG, HRP-linked & Cell Signaling Technology & $\# 7074$ \\
CAR & ABclonal & A1970 \\
ERK1/2 & Cell Signaling Technology & $\# 9102$ \\
Phospho-ERK1/2 & Cell Signaling Technology & $\# 9101$ \\
GAPDH & Santa Cruz Biotechnology & sc-47724 \\
HDAC-1 & Santa Cruz Biotechnology & sc-6298 \\
Histone 3 & ABclonal & A2352 \\
P38 & Cell Signaling Technology & $\# 9212$ \\
Phospho-P38 & Cell Signaling Technology & $\# 9211$ \\
Tubulin & Sigma-Aldrich & T9026 \\
UGT1A1 & Abcam & Ab170858 \\
& & \\
\hline
\end{tabular}

(Fig. 3A). Furthermore, Western blot analysis of CYP2B10 was inconclusive, with no apparent detection in either vehicle- or $\mathrm{Cd}^{2+}$. treated mice. Given that basal expression of CYP2B10 in neonatal intestinal tissue is low, CAR activation was assayed more directly via nuclear localization.

Intestinal tissues taken from $h U G T 1 / \mathrm{Nrf}^{+/-}$and $h U G T 1 / \mathrm{Nrf} 2^{-/-}$ neonates exposed to $\mathrm{Cd}^{2+}$ were fractionated and examined for nuclear CAR accumulation by Western blot analysis (Fig. 3, B and C). There was a statistically significant increase in the nuclear accumulation of CAR in $h U G T 1 / \mathrm{Nrf}^{+/-}$(3.7-fold) and in hUGT1/Nrf2 $2^{-/-}$mice (1.8fold) following exposure to $\mathrm{Cd}^{2+}$. This localization can occur independently of NRF2 but is 2-fold less in $h U G T 1 / N r f 2^{-1-}$ compared with $h U G T 1 / \mathrm{Nrf}^{+/-}$neonatal mice. This indicates that NRF2 is not responsible for the activation of CAR by $\mathrm{Cd}^{2+}$.

In Vivo $\mathrm{Cd}^{2+}$ Exposure Induces Mitogen-Activated Protein Kinase Phosphorylation and CAR Localization. The regulation of CAR localization makes use of extracellular signal-regulated kinases 1 and 2 (ERK1/2) and mitogen-activated protein kinase 11 (P38) phosphorylation, which was examined by Western blot analysis. Neonatal $h U G T 1$ mice were treated orally with a single dose of $\mathrm{Cd}^{2+}$ $(10 \mathrm{mg} / \mathrm{kg})$ or water, and intestinal tissues were collected $30,60,120$, and 240 minutes after exposure. Following treatment, the neonatal mice experienced dramatic changes in mitogen-activated protein kinase (MAPK) phosphorylation: A large increase in cytoplasmic phosphorylated ERK1/2 occurred in intestinal tissue between 0 and 30 minutes after oral treatment before decaying (Fig. 4B). Additionally, there was an accumulation of P38 and phosphorylated-P38 in the nucleus (Fig. 4A). Similar phosphorylation patterns were observed in adult mice (data not shown). Consistent increases in CAR accumulation in the nucleus were observed in neonatal mice after 2 hours (Fig. 4C), coinciding with the dephosphorylation of ERK1/2 and the nuclear accumulation of P38. The observed changes in MAPK activation are consistent with known regulatory mechanisms for CAR by ERK (Koike et al., 2007) and P38 (Hori et al., 2016), supporting the observation that $\mathrm{Cd}^{2+}$ exposure activates intestinal CAR. The increased oxidative state following $\mathrm{Cd}^{2+}$ treatment, the activation of MAPK pathways, and the commensurate nuclear accumulation of CAR underlies the induction of intestinal UGT1A1.

PEITC Induction of UGT1A1 Retains CAR-Dependency Pattern in $\boldsymbol{h U G T 1 / N r f 2 ^ { - / - }}$ Mice. The observation of CAR-dependent regulation of hepatic UGT1A1 by pro-oxidants was observed in neonatal $h U G T 1 / \mathrm{Car}^{-/-}$mice treated with PEITC (Yoda et al., 2017). 


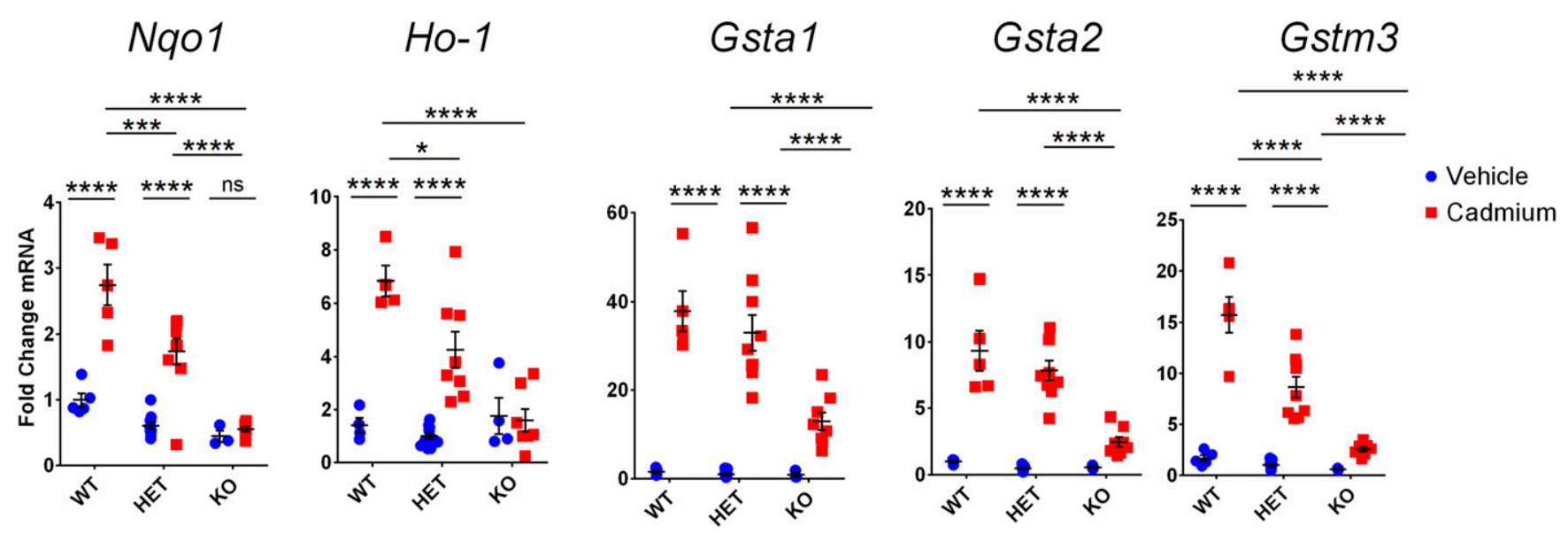

Fig. 1. NRF2-dependency of antioxidant response genes in neonatal $h U G T 1$ mice. Ten-day-old $h U G T 1 / N r f 2^{+/+}, h U G T 1 / N r f 2^{+/-}$, and $h U G T 1 / N r f 2^{-/-}$neonatal mice were treated with vehicle (blue circles) or $\mathrm{Cd}^{2+}(10 \mathrm{mg} / \mathrm{kg}$ once a day by mouth) (red squares) for 2 days before collection of intestinal tissue for RT-qPCR analysis of oxidative stress markers. The genes examined include Nqo1, Ho-1, Gstal, Gsta2, and Gstm3. (Mean \pm S.E.M., two-way ANOVA; $* P<0.05 ; * * * P<0.001 ; * * * * P<0.0001$ ) WT, wild-type; HET, heterozygous; KO, knockout.

The tissue-specific induction patterns of hepatic and intestinal UGTIA1 and Cyp $2 b 10$ genes were observed in the $h U G T 1 / \mathrm{Nrf} 2^{-/-}$neonatal mice. The increased clearance of bilirubin in neonates treated with PEITC occurred concomitantly with increases in liver and intestinal expression of UGT1A1 and Cyp2b10 (Fig. 5). The inducibility of intestinal UGT1A1 by PEITC was reduced in $h U G T 1 / N r f 2^{-1-}$ (16.2-fold) mice compared with $h U G T 1 / \mathrm{Nrf}^{+/-}$mice (30.3-fold) (Fig. 5). No difference was observed in liver UGT1Al induction between $h U G T 1 / \mathrm{Nrf} 2^{+/-}$and $h U G T 1 / \mathrm{Nrf}^{-/-}$ neonates and the induction of CYP2B10 RNA was greatest in the liver (Fig. 5). These observations are consistent with the previous report on CAR dependency of hepatic UGT1A1 induction and CAR-independent intestinal UGT1A1 induction by PEITC (Yoda et al., 2017).

\section{Discussion}

It has been previously demonstrated that ITCs are capable of CARdependent induction of liver UGT1A1 (Yoda et al., 2017). It is well

established that NRF2 is the master regulator of the antioxidant response and can induce UGT1A1 (Yueh and Tukey, 2007). Nuclear receptors are susceptible to high levels of mutual regulation (Ratman et al., 2013) from shared protein interaction partners or through binding interactions at regulatory elements; therefore, it was hypothesized that oxidative stress could be coactivating CAR through NRF2. The role of oxidative stress in the regulation of NRF2 and CAR target genes in $h U G T 1 / \mathrm{Nrf}^{-/-}$mice was investigated. As anticipated, NRF2deficient mice do not generate the antioxidant response when challenged with $\mathrm{Cd}^{2+}$. However, the $h U G T 1 / N r f 2^{+/-}$mice exhibited a diminished antioxidant response in comparison with wild-type mice, suggesting haploinsufficiency.

By breeding $N r 2^{-/-}$mice with $h U G T 1$ mice, pro-oxidants could be screened for their ability to reduce TSB levels and examine the impact of this action in an NRF2-dependent fashion. The NRF2-KEAP1 pathway is not required for $\mathrm{Cd}^{2+}$ and ITCs to induce intestinal UGT1A1 in hUGT1 mice to reduce TSB levels. This supports the hypothesis that

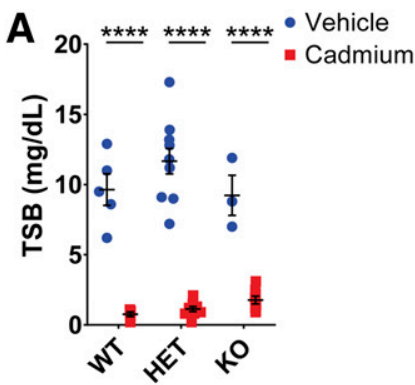

Nrf2 Genotype

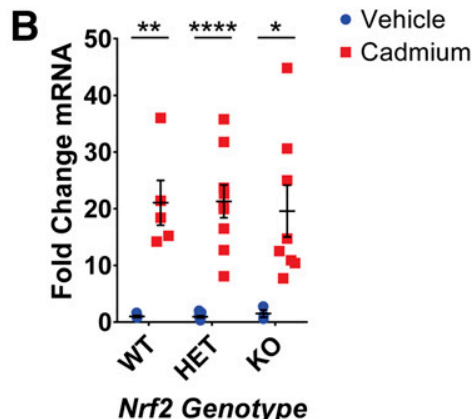

D

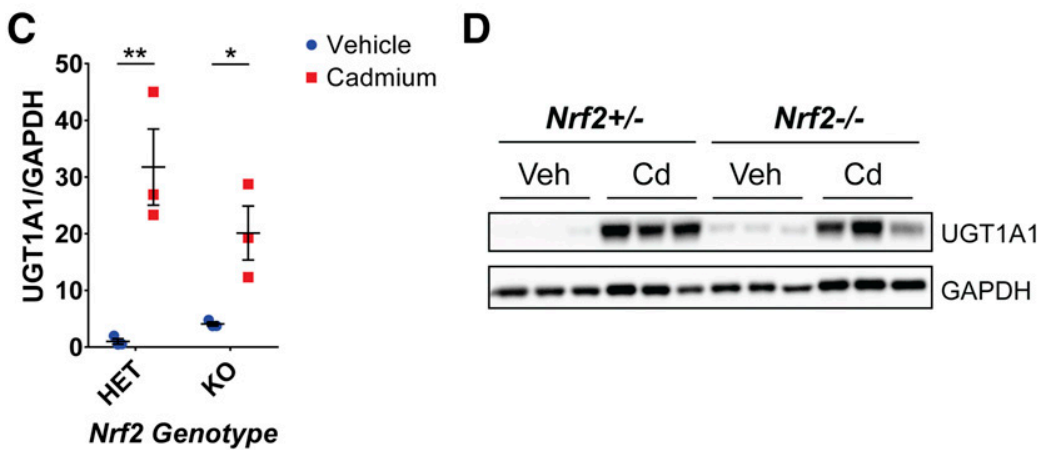

Fig. 2. $\mathrm{Cd}^{2+}$ reduces TSB levels in $h U G T 1$ neonatal mice in an NRF2-independent fashion. Ten-day-old $h U G T 1 / \mathrm{Nrf}^{+/+}$, $h U G T 1 / \mathrm{Nrf}^{+/-}$, and $h U G T 1 / \mathrm{Nrf}^{-/-}$neonatal mice were treated with vehicle (blue circles) or $\mathrm{Cd}^{2+}(10 \mathrm{mg} / \mathrm{kg}$ once a day by mouth) (red squares) for 2 days before collection of blood and intestinal tissues. (A) Changes in TSB levels $(\mathrm{mg} / \mathrm{dl})$ from mice treated with $\mathrm{Cd}^{2+}$. (B) Changes in the expression of the UGT1A1 gene. (C) Changes in the expression of the UGT1A1 protein as visualized by Western blot analysis and quantified by normalizing to GAPDH expression and set relative to vehicle-treated $h U G T 1 / \mathrm{Nrf}^{+/-}$mice. (Mean \pm S.E.M., two-way ANOVA; $* P<0.05$; $* * P<0.01$; $* * * * P<0.0001) \mathrm{WT}$, wild-type; HET, heterozygous; KO, knockout. 


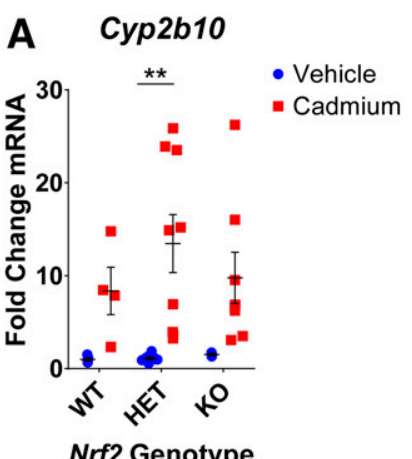

B Nuclear CAR

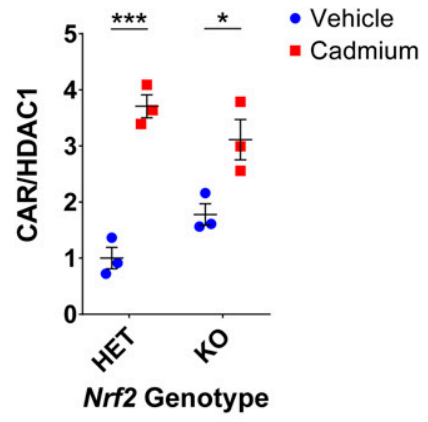

C

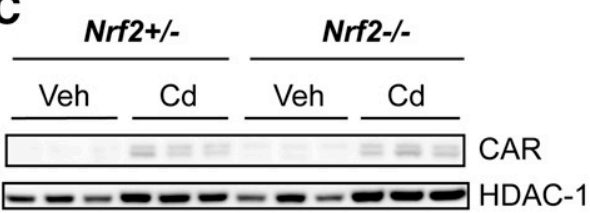

Fig. 3. NRF2-indendent changes of intestinal Cyp $2 b 10$ gene expression and CAR localization. Ten-day-old $h U G T 1 / N r f 2^{+/+}, h U G T 1 / N r f 2^{+/-}$, and $h U G T 1 / N r f 2^{-/-}$ neonatal mice were treated with vehicle (blue circles) or $\mathrm{Cd}^{2+}(10 \mathrm{mg} / \mathrm{kg}$ once a day by mouth) (red squares) for 2 days before collection of intestinal tissues. (A) Changes in the expression of the Cyp2b10 gene were observed by RT-qPCR. (B) Nuclear localization of CAR was determined by Western blot analysis (C) of fractionated intestinal protein extracts. Expression of CAR was quantified by normalizing to HDAC-1 and set relative to vehicle-treated $h U G T 1 / \mathrm{Nrf}^{+/-}$mice. (Mean \pm S.E.M., two-way ANOVA; $* P<0.05 ; * * P<0.01 ; * * * P<0.001$ )

CAR can be activated by oxidative stress (Yoda et al., 2017). In analysis of NR activity, we observed suppression of target genes for PXR and PPAR $\alpha$ and slight induction of the AhR target gene, Cyplal, in wildtype mice (Supplemental Fig. 1). The reason for the suppression is unclear, but the latter induction could have resulted from coregulation of Cyplal by NRF2 and the AhR (Shin et al., 2007). The loss of this induction in the heterozygous mice can be linked to haploinsufficiency of NRF2, and there was little change in the $N r f 2^{-1-}$ mice. From the examined NRs, there was evidence for CAR's activation from induction of the Cyp $2 b 10$ gene. Attempts were made to confirm protein induction of CYP2B10, but the expression levels were too low for detection. Subsequently, CAR's activation was assayed more directly. In the absence of a readily available antibody for phosphorylated CAR, fractionated intestinal samples from neonates treated with $\mathrm{Cd}^{2+}$ were analyzed for CAR nuclear localization. After an acute cadmium exposure, CAR localized to the nucleus in an NRF2-independent manner, which indicates that coactivation of CAR by NRF2 is not responsible for the induction of intestinal UGT1A1 and the reduction of TSB levels.

The increase in ERK1/2 phosphorylation is a confounding factor in the mechanism of CAR's activation, given that p-ERK1/2 inhibits the activation of p-Thr38-CAR, keeping it sequestered in the cytoplasm (Osabe and Negishi, 2011). However, it has been reported that in cardiac ventricular myocytes, ERK1/2 can be dephosphorylated by protein phosphatase 2 activity that is dependent on the activation of P38 (Liu and Hofmann, 2004). Furthermore, the phosphorylation status of ERK1/2 and $\mathrm{P} 38$ can be increased by $\mathrm{H}_{2} \mathrm{O}_{2}$ and $\mathrm{As}^{3+}$, respectively, both of which are linked to oxidative stress. The observation of a consistent nuclear accumulation of CAR during the decay of the p-ERK signal at the 2- and 4-hour time points suggests the reduction in ERK1/2 activity is sufficient to permit CAR activation, which occurs concurrently with the nuclear accumulation of P38/p-P38. In order for CAR to translocate to the nucleus, it must first be dephosphorylated by protein phosphatase 2 (Yoshinari et al., 2003) to interact with P38 to increase CAR's transactivation activity (Hori et al., 2016). Activated P38 phosphorylates
CAR at the conserved Thr38 residue to export CAR from the nucleus. These changes in the phosphorylation status of P38 and ERK1/2 were also observed in whole-cell lysate from a $h U G T 1$ adult time course (data not shown), supporting our hypothesis of the oxidative stress-MAPKCAR axis.

It has been reported that intestinal differentiation and crypt formation involves ROS and the activation of P38 (Houde et al., 2001; RodríguezColman et al., 2017). Intestinal maturation is essential to both nutrient uptake and metabolic waste/toxicant removal. From studies in hUGT1 mice, early intestinal expression of UGT1A1 is important for the metabolism of bilirubin while hepatic UGT1A1 expression is developmentally delayed (Fujiwara et al., 2010). Maturation of intestinal epithelial cells can be estimated by the decreases in genes related to cell stemness and the increase of functional genes related to the differentiated cell types. For example, the brush border glucosidase known as sucrose isomaltase, SIS, is only expressed in maturing and mature duodenum and jejunum enterocytes (Rubino et al., 1964). Alternatively, the $\operatorname{Lrp} 2$ gene encodes for a protein known as megalin, which mediates nutrient uptake by endocytosis, and its expression in the jejunum and ileum is downregulated as those tissues develop (Vázquez-Carretero et al., 2014). These and other genes, like Glb1 and $\mathrm{Krt20}$, are markers for intestinal maturation when down- and upregulated, respectively (Chen et al., 2017). Intestinal maturation markers were examined and it was determined that low doses of $\mathrm{Cd}^{2+}$ may induce intestinal maturation as inferred by inhibition of the Glbl and Lrp 2 genes and the induction of the Sis and Krt2O genes (Supplemental Fig. 3). Recently, it was reported that the nuclear receptor corepressor 1 (NCoR1), which regulates CAR activity at the PBREM (Mäkinen et al., 2002), is also capable of regulating intestinal
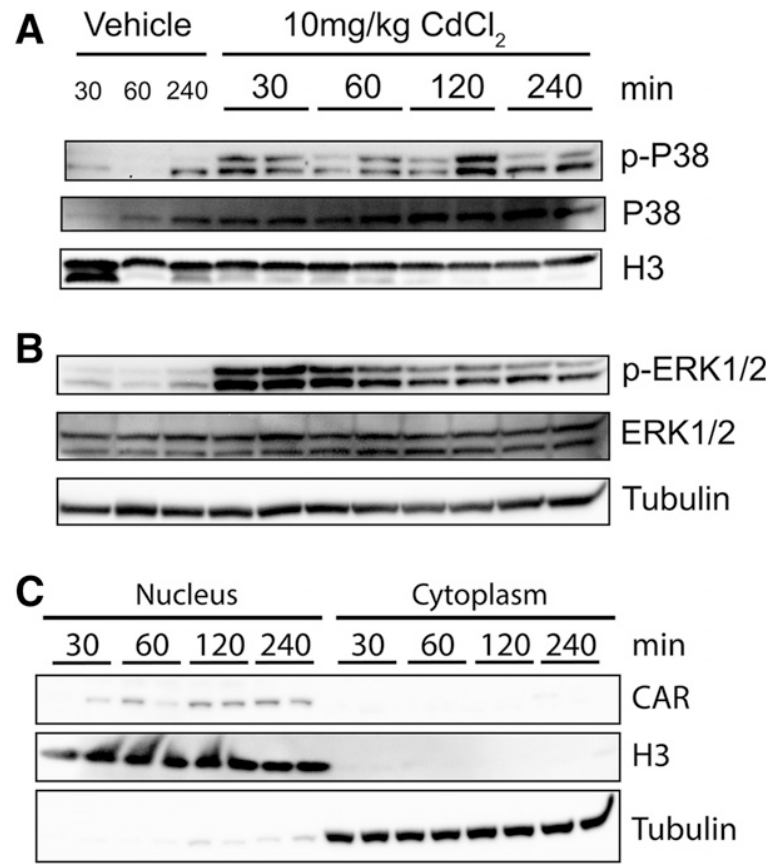

Fig. 4. Changes in intestinal MAPK phosphorylation and CAR localization following $\mathrm{Cd}^{2+}$ treatment in $h U G T 1$ neonates. Twelve-day-old neonatal $h U G T 1$ mice were treated with vehicle or $\mathrm{Cd}^{2+}(10 \mathrm{mg} / \mathrm{kg}$ by mouth $)$ and intestinal tissues were collected $30,60,120$, and 240 minutes after exposure. Intestinal protein extracts were fractionated into nuclear and cytosolic components, and changes in (A) nuclear P38 content and phosphorylation status, (B) cytosolic ERK1/2 content and phosphorylation status, and (C) CAR localization were visualized by Western blot analysis with $\mathrm{H} 3$ and tubulin for nuclear and cytoplasmic loading controls. Quantifications and statistical analyses for $(\mathrm{A}-\mathrm{C})$ can be found in Supplemental Figs. 4-6, respectively. 


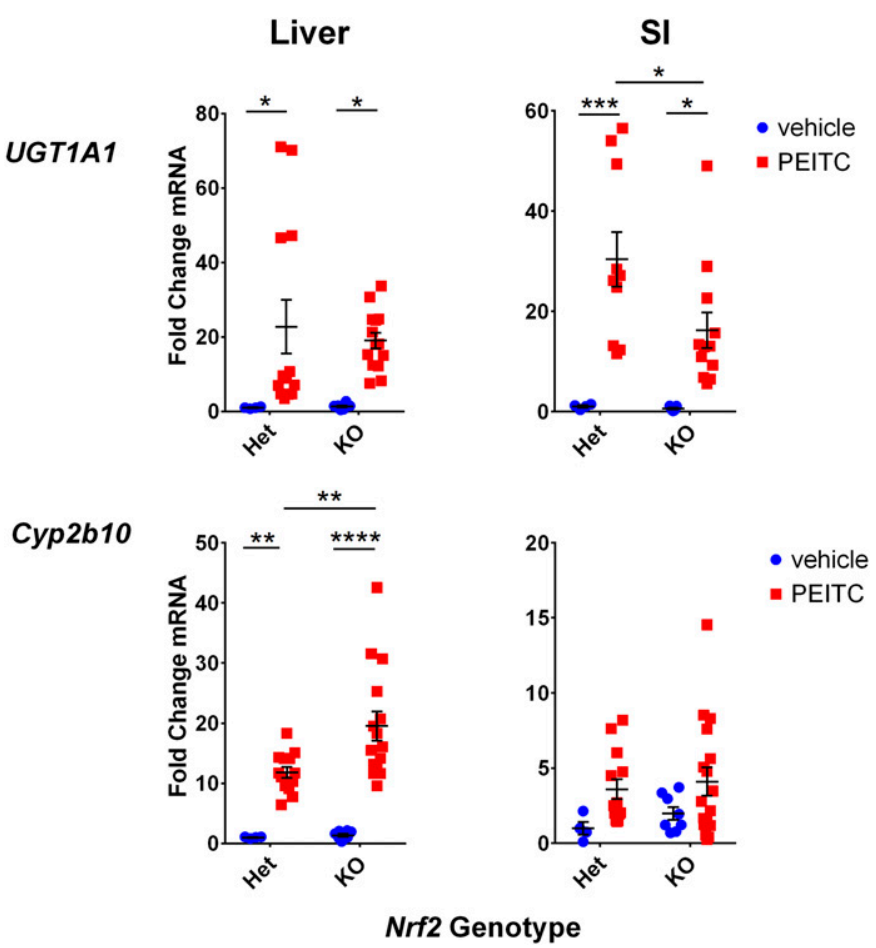

Fig. 5. Hepatic and intestinal induction of UGTIAl and Cyp2b10 in hUGT1/Nrf2 neonatal mice. Ten-day-old $h U G T 1 / N r f 2^{+/-}$and $h U G T 1 / N r f 2^{-1-}$ neonates were treated with vehicle (blue circles) or PEITC $(200 \mathrm{mg} / \mathrm{kg}$ once a day by mouth) (red squares) for 2 days before collection of liver and small intestine. Gene expression for UGTIAl and Cyp $2 b 10$ in each tissue was examined by RT-qPCR. (Mean \pm S.E.M., two-way ANOVA; $* P<0.05$; $* * P<0.01 ; * * * P<0.001$; **** $P<0.0001)$ HET, heterozygous; KO, knockout.

maturation (Chen et al., 2017). Intestinal epithelial cell-specific knockout of NCoR1 leads to changes in maturation markers and induction of UGT1A1 similar to what we have observed. Thus, it may be possible that $\mathrm{Cd}^{2+}$ cadmium inhibits NCoR1, which increases maturation and derepresses CAR's transcriptional activity.

The most obvious implication of the oxidative stress-MAPK-CAR signaling axis resides in changes in xenobiotic metabolism from prooxidant activation of CAR. This could impact therapeutic outcomes in patients exposed to environmental pro-oxidants, such as heavy metals from smoking or contaminated drinking water and soil, or from indirect antioxidants found in certain diets and commercial supplements. It is possible that future studies making use of RNA-seq, metabolomics, and exposure data may reveal a correlation between pro-oxidant, toxicant exposure, and an individual variability in xenobiotic metabolism stemming from CAR activity.

\section{Authorship Contributions}

Participated in research design: Paszek, Tukey.

Conducted experiments: Paszek.

Performed data analysis: Paszek.

Wrote or contributed to the writing of the manuscript: Paszek, Tukey.

\section{References}

Bonzo JA, Bélanger A, and Tukey RH (2007) The role of chrysin and the ah receptor in induction of the human UGT1A1 gene in vitro and in transgenic UGT1 mice. Hepatology 45:349-360. Bosma PJ, Seppen J, Goldhoorn B, Bakker C, Oude Elferink RP, Chowdhury JR, Chowdhury NR, and Jansen PL (1994) Bilirubin UDP-glucuronosyltransferase 1 is the only relevant bilirubin glucuronidating isoform in man. J Biol Chem 269:17960-17964.

Burchell B, Coughtrie M, Jackson M, Harding D, Fournel-Gigleux S, Leakey J, and Hume R (1989) Development of human liver UDP-glucuronosyltransferases. Dev Pharmacol Ther $\mathbf{1 3}$ $70-77$.

Chen S, Lu W, Yueh MF, Rettenmeier E, Liu M, Paszek M, Auwerx J, Yu RT, Evans RM, Wang $\mathrm{K}$, et al. (2017) Intestinal NCoR1, a regulator of epithelial cell maturation, controls neonatal hyperbilirubinemia [published correction appears in Proc Natl Acad Sci USA (2017) 114:E4115]. Proc Natl Acad Sci USA 114:E1432-E1440.

Chen S and Tukey RH (2018) Humanized UGT1 mice, regulation of UGT1A1, and the role of the intestinal tract in neonatal hyperbilirubinemia and breast milk-induced jaundice. Drug Metab Dispos 46:1745-1755.

Chen S, Yueh MF, Bigo C, Barbier O, Wang K, Karin M, Nguyen N, and Tukey RH (2013) Intestinal glucuronidation protects against chemotherapy-induced toxicity by irinotecan (CPT11). Proc Natl Acad Sci USA 110:19143-19148.

Chen S, Yueh MF, Evans RM, and Tukey RH (2012) Pregnane-x-receptor controls hepatic glucuronidation during pregnancy and neonatal development in humanized UGT1 mice. Hepatology 56:658-667.

Fujiwara R, Nguyen N, Chen S, and Tukey RH (2010) Developmental hyperbilirubinemia and CNS toxicity in mice humanized with the UDP glucuronosyltransferase 1 (UGT1) locus. Proc Natl Acad Sci USA 107:5024-5029.

Honkakoski P, Moore R, Gynther J, and Negishi M (1996) Characterization of phenobarbitalinducible mouse Cyp $2 \mathrm{~b} 10$ gene transcription in primary hepatocytes. J Biol Chem 271:9746-9753.

Hori T, Moore R, and Negishi M (2016) p38 MAP kinase links CAR activation and inactivation in the nucleus via phosphorylation at threonine 38. Drug Metab Dispos 44:871-876.

Houde M, Laprise P, Jean D, Blais M, Asselin C, and Rivard N (2001) Intestinal epithelial cell differentiation involves activation of $\mathrm{p} 38$ mitogen-activated protein kinase that regulates the homeobox transcription factor CDX2. J Biol Chem 276:21885-21894.

Koike C, Moore R, and Negishi M (2007) Extracellular signal-regulated kinase is an endogenous signal retaining the nuclear constitutive active/androstane receptor (CAR) in the cytoplasm of mouse primary hepatocytes. Mol Pharmacol 71:1217-1221.

Liu M, Chen S, Yueh MF, Fujiwara R, Konopnicki C, Hao H, and Tukey RH (2016) Cadmium and arsenic override NF-kB developmental regulation of the intestinal UGT1A1 gene and control of hyperbilirubinemia. Biochem Pharmacol 110-111:37-46.

Liu Q and Hofmann PA (2004) Protein phosphatase 2A-mediated cross-talk between p38 MAPK and ERK in apoptosis of cardiac myocytes. Am J Physiol Heart Circ Physiol 286: H2204-H2212.

Mäkinen J, Frank C, Jyrkkärinne J, Gynther J, Carlberg C, and Honkakoski P (2002) Modulation of mouse and human phenobarbital-responsive enhancer module by nuclear receptors. Mol Pharmacol 62:366-378.

McMahon M, Itoh K, Yamamoto M, Chanas SA, Henderson CJ, McLellan LI, Wolf CR, Cavin C, and Hayes JD (2001) The Cap'n'Collar basic leucine zipper transcription factor Nrf2 (NFE2 p45-related factor 2) controls both constitutive and inducible expression of intestinal detoxification and glutathione biosynthetic enzymes. Cancer Res 61:3299-3307.

Moi P, Chan K, Asunis I, Cao A, and Kan YW (1994) Isolation of NF-E2-related factor 2 (Nrf2), a NF-E2-like basic leucine zipper transcriptional activator that binds to the tandem NF-E2/AP1 repeat of the beta-globin locus control region. Proc Natl Acad Sci USA 91:9926-9930.

Mutoh S, Sobhany M, Moore R, Perera L, Pedersen L, Sueyoshi T, and Negishi M (2013) Phenobarbital indirectly activates the constitutive active androstane receptor (CAR) by inhibition of epidermal growth factor receptor signaling. Sci Signal 6:ra31

Osabe M and Negishi M (2011) Active ERK1/2 protein interacts with the phosphorylated nuclear constitutive active/androstane receptor (CAR; NR1I3), repressing dephosphorylation and sequestering CAR in the cytoplasm. J Biol Chem 286:35763-35769.

Pearson HA (1967) Life-span of the fetal red blood cell. J Pediatr 70:166-171.

Ratman D, Vanden Berghe W, Dejager L, Libert C, Tavernier J, Beck IM, and De Bosscher K (2013) How glucocorticoid receptors modulate the activity of other transcription factors: a scope beyond tethering. Mol Cell Endocrinol 380:41-54.

Rodríguez-Colman MJ, Schewe M, Meerlo M, Stigter E, Gerrits J, Pras-Raves M, Sacchetti A, Hornsveld M, Oost KC, Snippert HJ, et al. (2017) Interplay between metabolic identities in the intestinal crypt supports stem cell function. Nature 543:424-427.

Rubino A, Zimbalatti F, and Auricchio S (1964) Intestinal disaccharidase activities in adult and suckling rats. Biochim Biophys Acta 92:305-311.

Senekeo-Effenberger K, Chen S, Brace-Sinnokrak E, Bonzo JA, Yueh MF, Argikar U, Kaeding J, Trottier J, Remmel RP, Ritter JK, et al. (2007) Expression of the human UGT1 locus in transgenic mice by 4-chloro-6-(2,3-xylidino)-2-pyrimidinylthioacetic acid (WY-14643) and implications on drug metabolism through peroxisome proliferator-activated receptor $\alpha$ activation. Drug Metab Dispos 35:419-427.

Shin S, Wakabayashi N, Misra V, Biswal S, Lee GH, Agoston ES, Yamamoto M, and Kensler TW (2007) NRF2 modulates aryl hydrocarbon receptor signaling: influence on adipogenesis. Mol Cell Biol 27:7188-7197.

Sugatani J, Kojima H, Ueda A, Kakizaki S, Yoshinari K, Gong QH, Owens IS, Negishi M, and Sueyoshi T (2001) The phenobarbital response enhancer module in the human bilirubin UDP-glucuronosyltransferase UGT1A1 gene and regulation by the nuclear receptor CAR. Hepatology 33:1232-1238.

Timsit YE and Negishi M (2014) Coordinated regulation of nuclear receptor CAR by CCRP/ DNAJC7, HSP70 and the ubiquitin-proteasome system. PLoS One 9:e96092.

Vázquez-Carretero MD, Palomo M, García-Miranda P, Sánchez-Aguayo I, Peral MJ, Calonge ML, and Ilundain AA (2014) Dab2, megalin, cubilin and amnionless receptor complex might mediate intestinal endocytosis in the suckling rat. J Cell Biochem 115:510-522.

Yoda E, Paszek M, Konopnicki C, Fujiwara R, Chen S, and Tukey RH (2017) Isothiocyanates induce UGT1A1 in humanized UGT1 mice in a CAR dependent fashion that is highly dependent upon oxidative stress. Sci Rep 7:46489.

Yoshinari K, Kobayashi K, Moore R, Kawamoto T, and Negishi M (2003) Identification of the nuclear receptor CAR:HSP90 complex in mouse liver and recruitment of protein phosphatase $2 \mathrm{~A}$ in response to phenobarbital. FEBS Lett 548:17-20.

Yueh MF, Bonzo JA, and Tukey RH (2005) The role of Ah receptor in induction of human UDPglucuronosyltransferase 1A1. Methods Enzymol 400:75-91.

Yueh MF, Chen S, Nguyen N, and Tukey RH (2017) Developmental, genetic, dietary, and xenobiotic influences on neonatal hyperbilirubinemia. Mol Pharmacol 91:545-553.

Yueh MF and Tukey RH (2007) Nrf2-Keap1 signaling pathway regulates human UGT1Al expression in vitro and in transgenic UGT1 mice. J Biol Chem 282:8749-8758.

Address correspondence to: Dr. Robert H. Tukey, Department of Pharmacology, University of California San Diego, 9500 Gilman Drive, La Jolla, California 920930722. E-mail: rtukey@ucsd.edu 